\title{
Schisandrae Fructus Reduces Symptoms of 4-Vinylcyclohexene Diepoxide-Induced Ovarian Failure in Mice
}

\author{
Dasom Shin, ${ }^{1}$ Jinhee Ha, ${ }^{1}$ Seong Bin Hong, ${ }^{2}$ Geun-Hyung Kang, \\ Deok-Sang Hwang, ${ }^{3}$ and Hyunsu Bae ${ }^{1}$ \\ ${ }^{1}$ Department of Science in Korean Medicine, Graduate School, Kyung Hee University, 26 Kyungheedae-ro, Dongdaemoon-gu, \\ Seoul 02447, Republic of Korea \\ ${ }^{2}$ Biomix Inc., Goyang, Gyeonggi-do, Republic of Korea \\ ${ }^{3}$ Department of Obstetrics and Gynecology, College of Korean Medicine, Kyung Hee University, Seoul, Republic of Korea
}

Correspondence should be addressed to Hyunsu Bae; hbae@khu.ac.kr

Received 3 February 2017; Accepted 20 April 2017; Published 11 May 2017

Academic Editor: Jairo Kennup Bastos

Copyright (C) 2017 Dasom Shin et al. This is an open access article distributed under the Creative Commons Attribution License, which permits unrestricted use, distribution, and reproduction in any medium, provided the original work is properly cited.

\begin{abstract}
Menopause is associated with a decrease in the level of sex hormones such as ovarian estradiol and progesterone and can cause various symptoms such as depression, hot flash, fatigue, heart palpitations, and headache. Furthermore, there is a risk of developing complications such as osteoporosis, cardiovascular diseases, Alzheimer's disease, and ovarian cancer. Schisandrae Fructus (SF) is widely used in Korean medicine as a cure for such complications. This study was conducted to evaluate the therapeutic effects of SF against menopause symptoms associated with follicle depletion caused by the industrial chemical 4-vinylcyclohexene diepoxide (VCD) in mice. VCD directly targets the preantral follicles. Mice were injected with VCD (160 mg/kg intraperitoneally) daily for 15 days and then with SF dosage 3 times/week for six weeks. To evaluate the effects of SF, body weight, tail skin temperature, uterine weight, lipid profile, and osteocalcin levels were measured. A decrease in body weight and tail skin temperature and an increase in uterine weight were observed upon SF treatment. Moreover, SF treatment significantly decreased total cholesterol, triglyceride, osteocalcin, and low-density lipoprotein levels and low-density/high-density lipoprotein ratio. These results suggest the potential use of SF in the treatment of menopausal symptoms in women.
\end{abstract}

\section{Introduction}

Menopause is a natural process experienced by women [1]. It is caused by cessation of estrogen production by the ovaries [2]. Symptoms include hot flashes, anxiety, depression, night sweating, insomnia, vaginal dryness, and joint pain $[2,3]$. Furthermore, complications such as osteoporosis, cardiovascular disease [4], Alzheimer's disease [5], and ovarian cancer [6] may follow. Hormone replacement therapy (HRT) is employed to ameliorate menopausal symptoms in many menopausal women. However, HRT is known to cause breast cancer, ovarian cancer, uterine cancer, and gallbladder diseases [7-9].

In a previous study, an ovariectomy model (OVX) and a chemical model [4-vinylcyclohexene diepoxide (VCD)] were used to study menopause in women [10]. The OVX model is a common surgically induced animal model for studying menopause and used in the field of aging, such as mechanisms for skeletal responses to estrogen deficiency $[11,12]$. In the OVX model, ovarian hormones are removed rapidly rather than the gradual decline that occurs in natural menopause and the symptom is similar to women who undergo surgical menopause [10]. A chemical model is developed for perimenopause and postmenopause by administrating the diepoxide metabolite, 4-vinylcyclohexene (VCD) [13]. VCD is industrially used as a cross linking agent for production of epoxy resins [10,14]. VCD exposure selectively accelerates the natural loss of small primordial and primary ovarian follicles of mice without affecting other tissues [10, $15,16]$. Most of women undergo natural menopause and only less than $13 \%$ of women undergo surgical menopause [17]. Therefore, VCD-induced ovarian failure is a useful tool to mimic human menopause and retains residual ovarian 
tissues. We used the chemical model as it is closely related to mimic human menopausal transition [1].

Schisandrae Fructus (SF), which belongs to the Magnoliaceae family, is well known in Korean medicine. SF comprises sweet (fruit skin), sour (pulp), bitter/pungent (seed core), and salty components (all parts) [18, 19]. According to previous studies, It can also be used as antioxidant [20], antitumor [21], and antiaging agent [22] and in the treatment of liver diseases [23]. In Korea, SF has been used in various herbal prescriptions for treating menopausal symptoms [18]. The objective of this study was to analyze the effects of SF on menopausal symptoms in VCD-induced ovarian failure mice model.

\section{Materials and Methods}

2.1. Chemicals and Reagents. VCD (94956) and $17 \beta$-estradiol (E2; E8875) were purchased from Sigma-Aldrich Korea (Seoul, South Korea). VCD was dissolved in sesame oil (160 mg/kg; S3547, Sigma-Aldrich) and E2 in EtOH (0.5 mg/kg).

2.2. Extraction of SF. The extract of SF was provided from Biomix (Goyang, Gyeonggi-do, Republic of Korea). A decoction of the traditional medicine was prepared by extracting it with $30 \% \mathrm{EtOH}$ for $3 \mathrm{~h}$. Moreover, the remains of the herbs and impurities were separated from the extracted liquid by a filtration separation process. After filtration and drying, extracts of SF were remained liquefied. All extracting processes of it were controlled by Biomix Inc. SF was carefully measured to prepare the necessary doses for each treatment group and then dissolved in distilled water for $30 \mathrm{~min}$ at a controlled room temperature. SF was orally administered to mice at 25,100, and $400 \mathrm{mg} / \mathrm{kg} /$ day for 6 weeks.

2.3. High-Performance Liquid Chromatographic Analysis of SF. Gomisin N was purchased from the National Development Institute of Korean Medicine. HPLC-grade acetonitrile was purchased from Daejung Inc. The compound structure is presented in Figure 1. The purity of this standard compound was determined to be greater than $97 \%$ by normalization of the peak areas detected by HPLC analysis. SF was dissolved in $20 \mathrm{ml}$ of methanol and sonicated for $30 \mathrm{~min}$. The solution was filtered through a $0.45-\mu \mathrm{m}$ syringe filter, and the filtrate was used as the test solution. Ten microliters of the test solution was injected into an HPLC system (Waters 2695 Alliance HPLC system; detector: Waters 996 PDA; column: Fortis C18 $5 \mu \mathrm{m} 250 \times 4.6 \mathrm{~mm})$. The analysis involved an isocratic elution of acetonitrile and $0.1 \%$ formic acid $(70 / 30, \mathrm{v} / \mathrm{v})$ over $40 \mathrm{~min}$ at a flow rate of $1 \mathrm{ml} / \mathrm{min}$.

2.4. Animal Experiments. Six-week-old C57BL/6 female mice were purchased from Orient Bio (Orient Bio, Seongnam, South Korea). The mice were group-housed ( $n=$ case) and allowed to acclimatize (object) for 1 week before treatment with VCD. Mice were randomly divided into five experimental groups ( $n=8 /$ group): negative control mice received an intraperitoneal (i.p.) injection of sesame oil daily for 15 days. VCD + phosphate-buffered saline (PBS) group, VCD $+17 \beta$-Estradiol (E2) group, and VCD + SF-treated groups
(SF-treated mice were divided into three groups according to concentration of SF: 25,100 , and $400 \mathrm{mg} / \mathrm{kg})$ : mice received an i.p. injection daily of VCD $(160 \mathrm{mg} / \mathrm{kg})$ for 15 days. PBS was used as a vehicle control for SF. After dosing with VCD, PBS and each treatment were orally administered thrice a week during 6 weeks. Two days after the last dose (day 65 ), mice were sacrificed by $\mathrm{CO}_{2}$ asphyxiation, and uterus and serum samples were collected for analyses. No mice died during the experiment. All the experiments and animal handling procedures in this study were approved by the Animal Experimental Ethics Committee of the Kyung Hee University [KHUASP (SE)-16-037].

2.5. Body Weight and Tail Skin Temperature Analysis. The body weight and tail skin temperature of the mice were measured once a week for 9 weeks. The body weight was measured at the end of the experiment on an analytical balance for comparison between groups. The tail skin temperature was monitored during the sleep cycle using an infrared thermometer (11092060722, TPI 383, Summit, Korea). Three measurements were obtained $10 \mathrm{~min}$ apart, and the average value was used as a single data point.

2.6. Osteocalcin Analysis. Fasting serum samples were collected and stored at $-80^{\circ} \mathrm{C}$ before analysis. The osteocalcin levels in the serum samples were determined using a commercial enzyme immunoassay kit (Takara Bio Inc., Seoul, Korea) according to the manufacturer's instructions. A 96well microtiter plate was coated with an anti-mouse OC monoclonal antibody. The standard and sample were then added and incubated for 1 hat room temperature. A second ary peroxidase-labeled anti-Gla-OC monoclonal antibody was then added and incubated for $1 \mathrm{~h}$. Finally, the plates were treated with the substrate solution (TMBZ) for $30 \mathrm{~min}$, and the reaction was stopped with the addition of the TMBZ stop solution (50 $\mu \mathrm{L}$ per well). Optical density of the samples was measured at $450 \mathrm{~nm}$ using a microplate reader (SoftMax PRO, version 3.1. software, CA, USA).

2.7. Serum Biochemistry Analysis. For serum biochemical analysis, the clotted blood samples were centrifuged for $10 \mathrm{~min}$ at $1,500 \mathrm{~g}$ to obtain serum samples, which were analyzed using an automated analyzer (7080, Hitachi Ltd., Tokyo, Japan). The serum biochemistry parameters, including total cholesterol, triglyceride, low-density lipoprotein (LDL), and high-density lipoprotein (HDL), were analyzed.

2.8. Statistical Analysis. Statistical analysis of the data was conducted using Prism 5 software (GraphPad Software Inc., San Diego, CA, USA). All the values are presented as mean \pm standard error of the mean (SEM). Statistical significance was determined by one-way ANOVA, followed by Tukey's test using randomly selected samples. A $p$ value of $<0.05$ was considered statistically significant.

\section{Results}

3.1. Identification of SF. SF was characterized using Gomisin $\mathrm{N}$ by HPLC analysis (Figure 1(c)). By considering the amount 
Gomisin A

Gomisin N

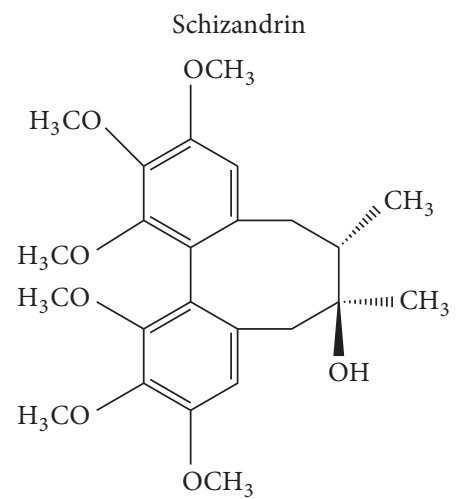<smiles>COc1cc2c(c(OC)c1OC)-c1c(cc3c(c1OC)OCO3)C[C@H](C)[C@](C)(O)C2</smiles>

(a)

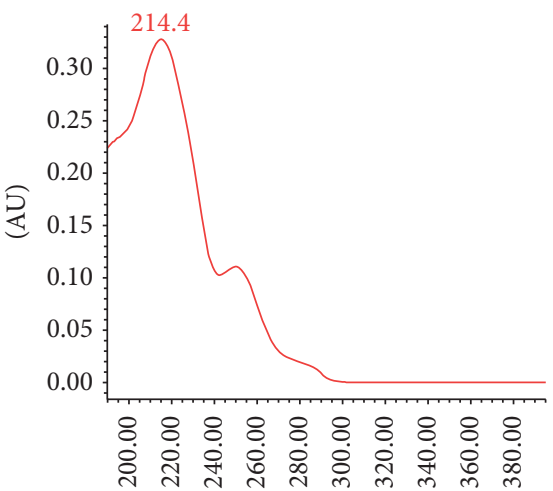

(nm)

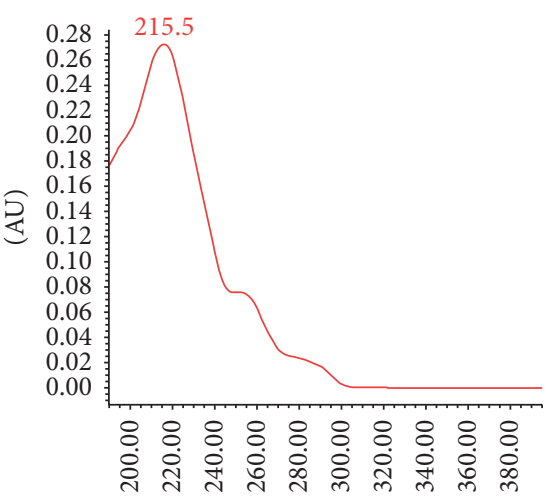

(nm)<smiles>COc1cc2c(c(OC)c1OC)-c1c(cc3c(c1OC)OCO3)C[C@@H](C)[C@H](C)C2</smiles>

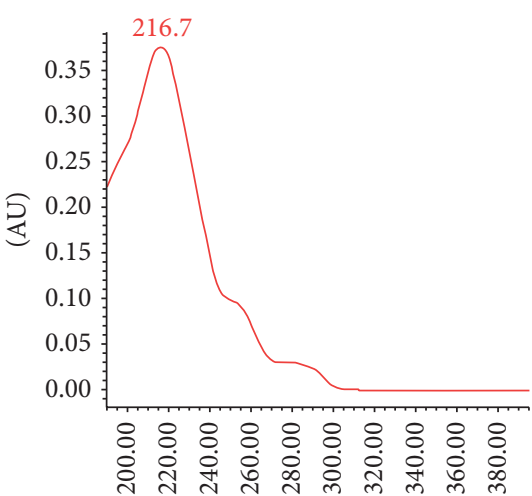

(b)

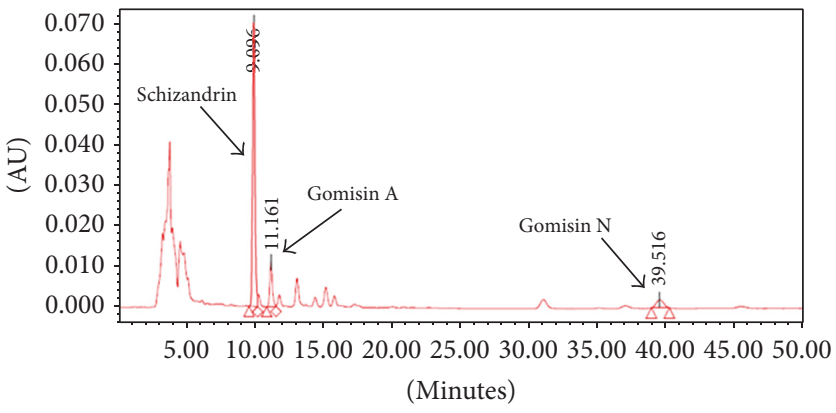

(c)

Figure 1: The structure of Schizandrin, Gomisin A, and Gomisin N (a), UV spectrum of Schizandrin, Gomisin A, and Gomisin N (b), and chromatogram of SF (c) using HPLC systems.

of Gomisin N in SF, a calibration curve was constructed with six concentration levels $(10.417,15.625,31.25,62.5,125$, and $250 \mu \mathrm{g} / \mathrm{ml}$ ). Linearity was evaluated using the correlation coefficient $\left(R^{2}\right)$ of the calibration curve. Gomisin $\mathrm{N}$ was identified at a retention time of $25.085 \mathrm{~min}$ and its UV spectrum is shown in Figure 1(b). According to quantitative analysis, the yield of SF was $902.833 \mu \mathrm{g} / \mathrm{g}$.

3.2. SF Influences Menopause-Related Changes in Lipid Profile. On day 65, we collected serum samples from all groups to investigate the lipid profile. Total cholesterol, LDL, and LDL/HDL levels were significantly higher in the VCD + Vehicle group than in the control mice (Figures 2(a), 2(c), and 2(e)). Triglyceride and HDL levels were higher, but not significantly, in the VCD + Vehicle group than in the control mice (Figures 2(b) and 2(d)). However, the SF-treated groups had significantly lower total cholesterol, triglyceride, LDL, and LDL/HDL levels than the VCD + Vehicle group did (Figures 2(a), 2(b), 2(c), and 2(e)). However, there was no significant difference in HDL levels between the groups (Figure 2(d)).

3.3. SF Is Associated with Menopause-Related Changes in Body Weight and Tail Skin Temperature. We observed body weight and tail skin temperature for 9 weeks. After dosing (day 15), a gradual difference in body weight and tail skin 


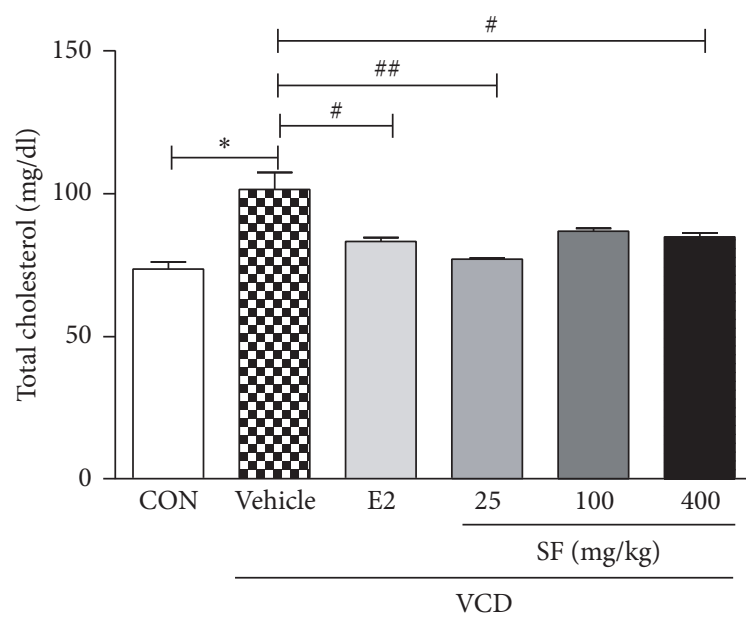

(a)

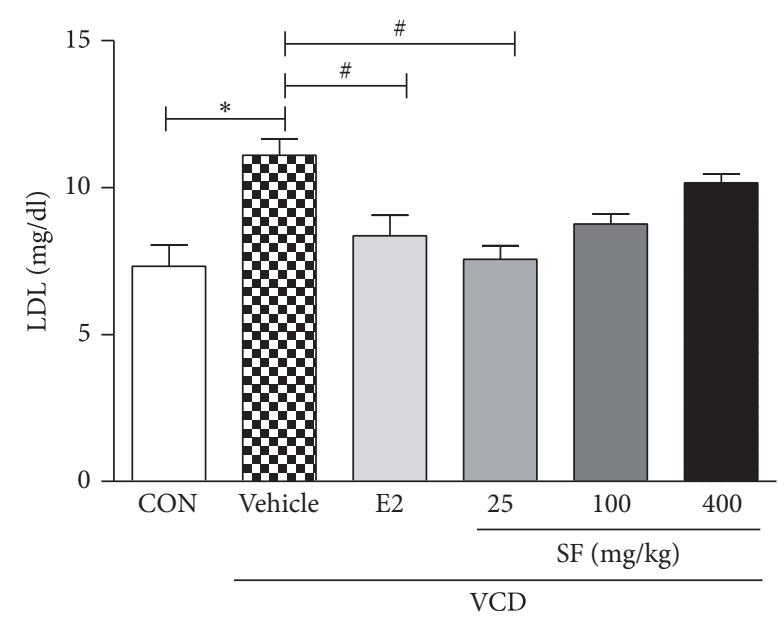

(c)

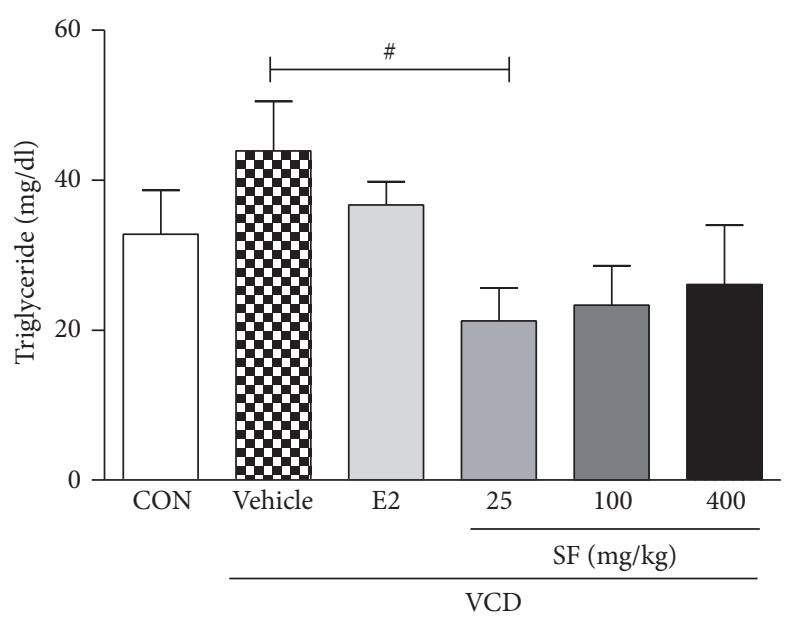

(b)

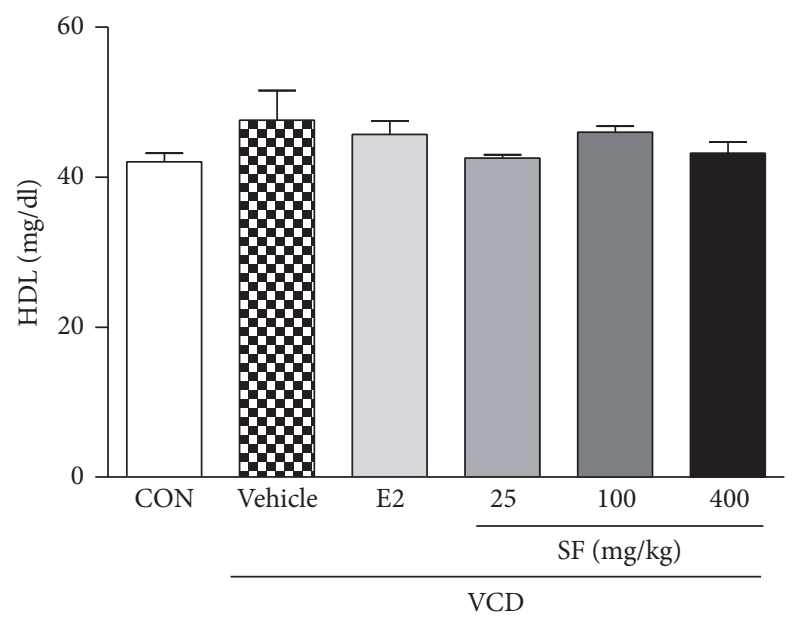

(d)

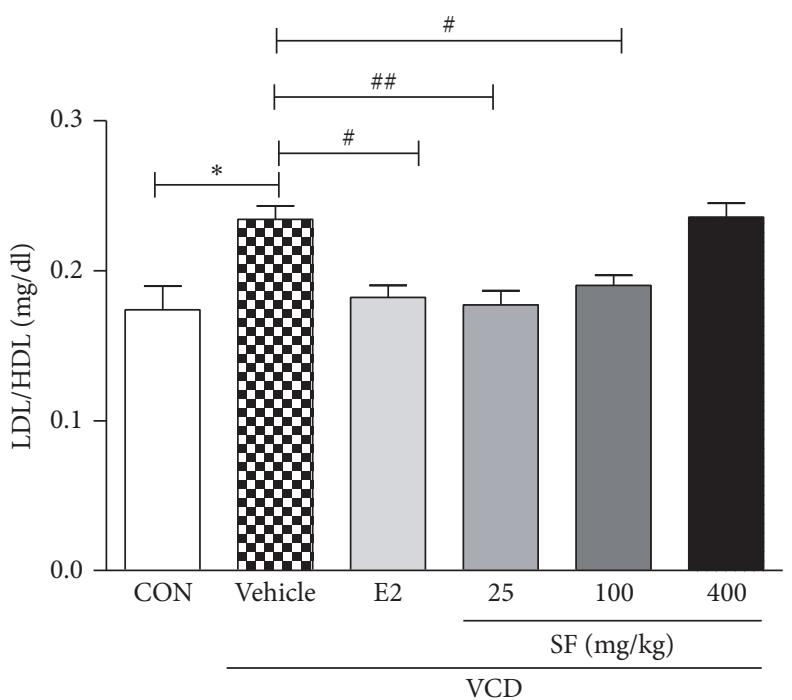

(e)

FIGURE 2: Effects of SF on changes in lipid profile. On day 65, serum was collected from all groups. (a) Total cholesterol, (b) triglyceride, (c) LDL, (d) HDL, and (e) LDL/HDL. Data are presented as mean \pm SEM. $p$ values are obtained using one-way analysis of variance (one-way ANOVA) by comparing the treatment group with VCD + Vehicle group. ${ }^{*} p<0.05$ compared with the CON group; ${ }^{\# \#} p<0.01$ and ${ }^{\#} p<0.05$ compared with the VCD + Vehicle group. 


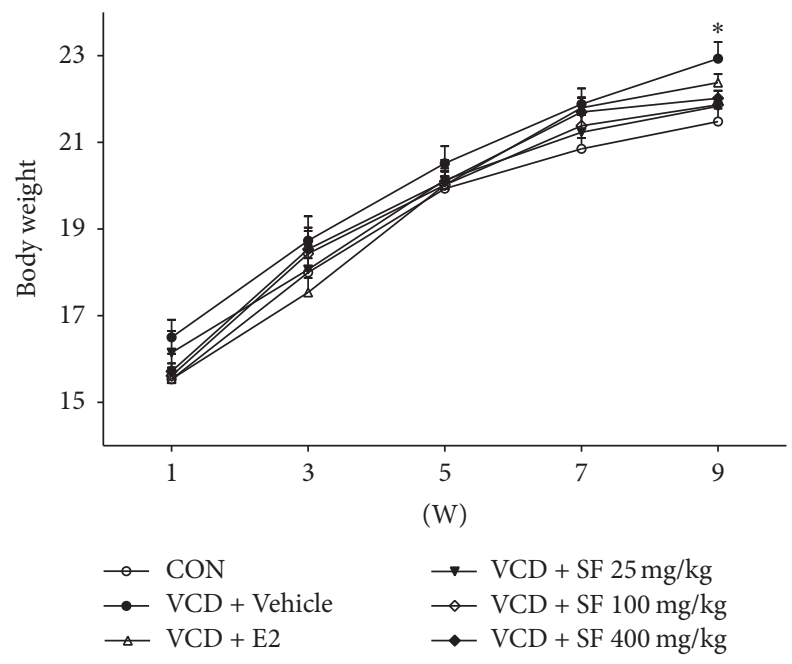

FIgURE 3: Time course of changes in body weight of mice. The body weight of mice was measured for 9 weeks. Data are presented as mean \pm SEM. Differences between treatments were analyzed by oneway ANOVA with Tukey post hoc analysis. ${ }^{*} p<0.05$ compared with the CON group.

temperature was observed in control mice and VCD-treated animals. From the eighth week, body weight of mice in the VCD group was significantly higher than that of control group mice (Figure 3$)$. Mice in the SF-treated groups $(25,100$, and $400 \mathrm{mg} / \mathrm{kg}$ ) exhibited decreased body weight compared to that reported for VCD + Vehicle group mice. However, there was no significant difference (Figure 3 ). On day 65, we measured the uterine weight. The uterine weights of mice in the VCD + Vehicle group were lower than those of control group mice were. However, the uterine weights of mice in the SF-treated groups ( $25 \mathrm{mg} / \mathrm{kg}$ and $400 \mathrm{mg} / \mathrm{kg}$ ) were higher than those of the VCD + Vehicle group mice were (Figure 4). The $100 \mathrm{mg} / \mathrm{kg}$ SF-treated group demonstrated a moderate increase compared to that reported for the VCD + Vehicle group. The tail skin temperatures were significantly higher in the VCD + Vehicle group than in those in the control group (Table 1). However, the temperatures in the VCD + E2 and SF-treated groups $(25,100$, and $400 \mathrm{mg} / \mathrm{kg})$ significantly decreased.

3.4. Inhibitory Effects of SF on Osteocalcin Production. Previous studies have demonstrated that osteoporosis is associated with menopause. After menopause, osteocalcin levels increase owing to the lack of sex hormones such as estrogen and androgen. Furthermore, osteocalcin is considered a bone turnover marker $[24,25]$. Therefore, we hypothesized that SF prevents the development of osteoporosis by reducing osteocalcin levels. Serum osteocalcin levels in the VCDtreated group were significantly higher than those in the control group were. However, osteocalcin levels in the SF treatment groups were lower than those in the VCD + Vehicle group were, particularly in the SF $25 \mathrm{mg} / \mathrm{kg}$ and SF $400 \mathrm{mg} / \mathrm{kg}$ groups (Figure 5).

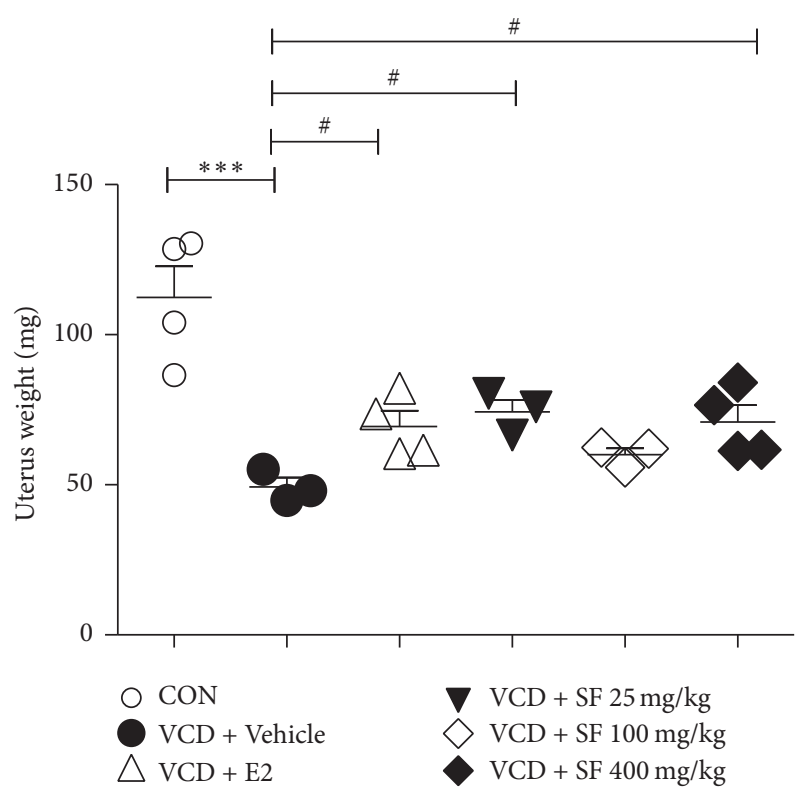

FIGURE 4: Effects of SF on changes in uterine weight. Uterine weight was measured at the end of the 9-week treatment period. Data are presented as mean \pm SEM. $p$ values are obtained using one-way analysis of variance (one-way ANOVA) by comparing the treatment group with VCD + Vehicle mice group. ${ }^{* * *} p<0.001$ compared with the CON group; ${ }^{*} p<0.05$ compared with the VCD + Vehicle group.

\section{Discussion}

Menopause is recognized by the cessation of menstrual cyclicity for at least one year [26, 27]. There are many factors that contribute to menopause, including age, health, environment, and lifestyle. This is caused by the depletion of germ cells and loss of ovarian follicular activity [6, 28]. In the previous report, authors suggested that impairment of BRCA1-related DNA double strand break (DSB) repair is associated with accelerated loss of ovarian follicular reserve and accumulation in mice and humans [29]. However, the exact mechanisms that cause menopause are still unknown. Menopause could lead to many diseases such as cardiovascular diseases [30], osteoporosis [31], ovarian cancer [8], and Alzheimer's disease [5]. The ovaries produce the sex steroids estrogen and progestins. However, as oocytes cannot be generated after birth, menopause occurs owing to the depletion of primordial follicles or ovarian failure [32, 33]. A clinical study reported some differences in symptoms of menopause between transitional hormone loss (natural menopause) and surgery. Compared with naturally occurring menopause, surgery-induced menopause was characterized by a lower memory score in women. The results suggested that surgery-induced menopause may have negative effects on brain function $[34,35]$. With age, most women develop various diseases owing to a decrease in hormonal levels induced by menopause [35]. Many studies have used ovariectomy (OVX) and chemical induction (VCD) to generate models for studying menopause. OVX, a surgical method for removing ovaries, ceases hormone secretion. This increases the risk of osteoporosis and decreases cognitive function in 
TABLE 1: Time course of changes in tail skin temperature of mice.

\begin{tabular}{lccccc}
\hline Tail skin temperature & 1 weeks & 3 weeks & 5 weeks & 7 weeks & 9 weeks \\
\hline CON & $22.64 \pm 0.22$ & $22.16 \pm 0.08$ & $22.42 \pm 0.17$ & $22.34 \pm 0.44$ & $23.00 \pm 0.13$ \\
VCD + Vehicle & $22.80 \pm 0.08$ & $23.23 \pm 0.08^{* *}$ & $23.63 \pm 0.16^{* *}$ & $23.42 \pm 0.19^{* *}$ & $23.27 \pm 0.17$ \\
VCD + E2 & $22.25 \pm 0.10$ & $22.98 \pm 0.20$ & $22.45 \pm 0.15^{\# \# \#}$ & $22.57 \pm 0.27^{\#}$ & $22.75 \pm 0.41$ \\
VCD + SF 25 mg/kg & $21.94 \pm 0.25^{\#}$ & $21.66 \pm 0.20^{\# \#}$ & $21.91 \pm 0.19^{\# \# \#}$ & $22.79 \pm 0.36$ & $22.36 \pm 0.27^{\#}$ \\
VCD + SF 100 mg/kg & $21.80 \pm 0.20^{\# \#}$ & $21.52 \pm 0.28^{\# \# \#}$ & $22.64 \pm 0.18^{\# \#}$ & $21.91 \pm 0.27^{\# \# \#}$ & $22.09 \pm 0.27$ \\
VCD + SF 400 mg/kg & $21.60 \pm 0.37^{\# \#}$ & $21.63 \pm 0.31^{\# \# \#}$ & $21.28 \pm 0.24^{\# \#}$ & $21.55 \pm 0.16^{\# \# \#}$ & $23.00 \pm 0.35$ \\
\hline
\end{tabular}

The tail skin temperature was measured at nine weeks. Data represent mean \pm SEM. ${ }^{* *} p<0.01$, compared with the CON group. ${ }^{\#} p<0.05$, ${ }^{\# \#} p<0.01$, and $\# p<0.001$ compared with the VCD + Vehicle group. Group and treatment differences were analyzed via one-way ANOVA with Tukey post hoc analysis.

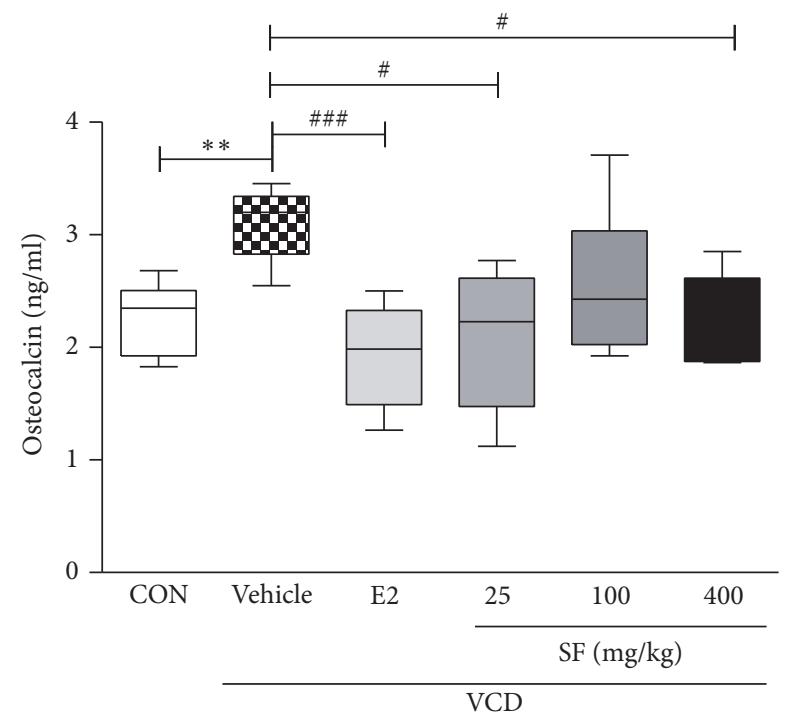

FIGURE 5: Effects of SF on changes in osteocalcin levels. On day 65, serum samples were collected from all groups. Data are presented as mean \pm SEM. $p$ values are obtained using one-way analysis of variance (one-way ANOVA) by comparing the treatment group with VCD + Vehicle mice. ${ }^{* *} p<0.01$ compared with the CON group; \#\#\# $p<0.001$ and $^{\#} p<0.05$ compared with the VCD + Vehicle group.

women $[11,35]$. On the other hand, VCD-induced follicle depletion mimics human menopausal transition [1]. VCD directly targets prenatal follicles and causes the loss of ovarian follicles in mice [16, 36, 37]. Furthermore, VCD can alter $17 \beta$-estradiol level and estrous cyclicity [37]. According to some previous studies $[10,14]$, VCD-induced ovarian failure mice model, rather than OVX-induced model, is similar to menopause in humans. Symptoms induced by VCD, such as estrous cyclicity and changes in LH, FSH, and estrogen levels and a decrease in uterine and ovarian weights, resemble naturally occurring menopausal symptoms in women. Furthermore, VCD became known as a potential risk of human exposure to environmental ovarian toxicants in women [33]. Therefore, we used the VCD-induced ovarian failure mice model to observe the expected menopausal changes in women.

In clinics, HRT is widely used for the treatment of menopause-inducing symptoms. This therapy can alleviate menopausal symptoms and protect women from diseases such as osteoporosis and cardiovascular diseases [38, 39]. According to a previous study, HRT reduced the risk of colorectal cancer and Alzheimer's disease [40]. However, as HRT is estrogen-based, it can induce many other diseases such as breast cancer [7, 41, 42], ovarian cancer [8], gallbladder disease [9], endometrial hyperplasia [43], coronary heart disease [44], and venous thrombosis [45]. To resolve these limitations, herbal medicine could be used as alternate therapeutic strategy in the treatment of the menopauseassociated diseases [46].

In many Asian countries, SF (Magnoliaceae family) is widely used in traditional medicine for the treatment of menopausal symptoms. SF is known as wu-wei-zi in Chinese [47], Gomishi in Japanese, and Omicha in Korea [48, 49]. It is associated with five flavors: sweet, sour, bitter, astringent, and salty flavors. Each of these flavors has various effects on different organs. Sour and salty components are believed to have good effects on the liver and testicles, bitter and stringent ones are believed to have good effects on the heart and lungs, and sweet component is believed to have good effects on the stomach [22]. In addition, numerous clinical trials have reported the efficacy of SF in conditions such as cough, dyspnea, dysentery, insomnia, amnesia, tumor, CNS, and cardiotonic disorder [18, 50, 51]. In Korea, SF is used in various herbal prescriptions for the treatment of menopausal symptoms, including Pheongbojinsimdan, Gamiondamtang, and Insugsan [18]. SF contains 13 lignans [52], including schisandrin [53] and Gomisin [54]. Among the ingredients of SF, schisandrin B (sch B) is the most abundant dibenzocyclooctadiene lignan, which is clinically used to treat hepatitis [55], cardiovascular diseases [53], and scopolamineinduced dementia [56]. According to a previous study, sch B can promote Treg proliferation, which secretes antiinflammatory cytokines [57]. Furthermore, increased Treg suppresses bone resorption and osteoclast differentiation in bone marrow. Some articles reported that osteoporosis in postmenopausal women could be prevented $[58,59]$. We also hypothesized that the administration of SF to VCD-induced ovarian failure mice could alleviate menopausal symptoms, such as an increase in body weight, tail skin temperature, and osteocalcin levels. Our present data demonstrated that the body weight of VCD group mice significantly increased after 8 weeks compared to that reported for the control group mice. Hot flashes are the most frequent symptoms of peri- and postmenopause period resulting from changing levels of estrogen in the body [60]. Hot flashes occur due to malfunction of the 
thermoregulatory system, which regulates body temperature and maintains homeostatic heat levels [61]. The underlying mechanism remains unknown. However, it is hypothesized that the rapid decline of estrogen at the time of menopause causes inducing hot flashes by influencing the action of nonepinephirine and serotonin. Tail skin temperature was also significantly elevated compared to that reported for the control group. Moreover, we observed a significant increase in uterine weight in the SF treatment groups compared to that in the VCD control group. It is well known that postmenopausal women have a high risk of weight gain [62] and type 2 diabetes [33, 63]. Because VCD is used to cause ovarian failure, uterine weight is typically decreased after VCD administration that is due to loss of tropic effects of ovarian estrogen on uterus. The reduction in uterine weight resulted from the withdrawal of ovarian estrogen and its wellknown tropic effects on this target tissue. We also found the reduction of uterine weight after VCD administration compared to the control. However, the uterine weight gains in the SF-treated groups were lower than those of the E2 group. Increased uterine weight is a typical adverse effect of E2 therapy, because it may cause uterine cell proliferation and possible tumor development. Further examination for the exact mechanisms of SF on uterine weight will be needed.

Osteoporosis, a disease with decreased bone strength and increase in the risk of a broken bone, generally develops after menopause in postmenopausal women [64, 65]. Some previous studies suggested that osteocalcin serves as a major factor in osteoblast differentiation and can be used as a potential predictor of diabetes in postmenopausal women $[66,67]$. Osteocalcin levels in the VCD treatment group were higher than those in the control group. However, a significant reduction in osteocalcin levels was observed in the SF treatment groups $(25 \mathrm{mg} / \mathrm{kg}$ and $400 \mathrm{mg} / \mathrm{kg}$ ). Based on these data, SF was suggested to effectively relieve various menopause-inducing symptoms in women.

\section{Conclusion}

Our data suggest that SF can successfully ameliorate menopausal symptoms in VCD-induced ovarian failure mice. SF could be used as a potential candidate for ameliorating symptoms of menopause in women.

\section{Conflicts of Interest}

The authors have no conflicts of interest to declare.

\section{Acknowledgments}

This work was supported by the Korea Science and Engineering Foundation (KOSEF) grant (no. 2009-0063466, HB) through the National Research Foundation of Korea, funded by the Ministry of Science, ICT, and Future Planning.

\section{References}

[1] S. V. Koebele and H. A. Bimonte-Nelson, "Modeling menopause: The utility of rodents in translational behavioral endocrinology research," Maturitas, vol. 87, pp. 5-17, 2016.
[2] M. H. Kim, Y. Y. Choi, J. M. Han et al., "Ameliorative effects of Schizandra chinensis on osteoporosis via activation of estrogen receptor (ER)- $\alpha /-\beta$," Food and Function, vol. 5, no. 7, pp. 15941601, 2014.

[3] H. Y. Chen, Y. H. Lin, J. C. Wu et al., "Prescription patterns of Chinese herbal products for menopausal syndrome: analysis of a nationwide prescription database," Journal of Ethnopharmacology, vol. 137, no. 3, pp. 1261-1266, 2011.

[4] S. E. Appt and K. F. Ethun, "Reproductive aging and risk for chronic disease: insights from studies of nonhuman primates," Maturitas, vol. 67, no. 1, pp. 7-14, 2010.

[5] K. M. Webber, G. Casadesus, M. W. Marlatt et al., "Estrogen bows to a new master: The role of gonadotropins in Alzheimer pathogenesis," Annals of the New York Academy of Sciences, vol. 1052, pp. 201-209, 2005.

[6] E. R. Smith, Y. Wang, and X.-X. Xu, "Development of a mouse model of menopausal ovarian cancer," Frontiers in Oncology, vol. 4, Article 36, 2014.

[7] C. Schairer, J. Lubin, R. Troisi, S. Sturgeon, L. Brinton, and R. Hoover, "Menopausal estrogen and estrogen-progestin replacement therapy and breast cancer risk," Journal of the American Medical Association, vol. 283, no. 4, pp. 485-491, 2000.

[8] B. Trabert, N. Wentzensen, H. P. Yang et al., "Ovarian cancer and menopausal hormone therapy in the NIH-AARP diet and health study," British Journal of Cancer, vol. 107, no. 7, pp. 11811187, 2012.

[9] E. Barrett-Connor and D. Grady, "Hormone replacement therapy, heart disease, and other considerations," Annual Review of Public Health, vol. 19, pp. 55-72, 1998.

[10] T. A. Van Kempen, T. A. Milner, and E. M. Waters, "Accelerated ovarian failure: a novel, chemically induced animal model of menopause," Brain Research, vol. 1379, pp. 176-187, 2011.

[11] L. E. Wright, P. J. Christian, Z. Rivera et al., "Comparison of skeletal effects of ovariectomy versus chemically induced ovarian failure in mice," Journal of Bone and Mineral Research, vol. 23, no. 8, pp. 1296-1303, 2008.

[12] S. C. Garner, J. J. B. Anderson, M.-H. Mar, and I. Parikh, "Estrogens reduce bone loss in the ovariectomized, lactating rat model," Bone and Mineral, vol. 15, no. 1, pp. 19-31, 1991.

[13] J. C. Lohff, P. J. Christian, S. L. Marion, and P. B. Hoyer, "Effect of duration of dosing on onset of ovarian failure in a chemicalinduced mouse model of perimenopause," Menopause, vol. 13, no. 3, pp. 482-488, 2006.

[14] P. B. Hoyer, P. J. Devine, X. Hu, K. E. Thompson, and I. G. Sipes, "Ovarian toxicity of 4-vinylcyclohexene diepoxide: a mechanistic model," Toxicologic Pathology, vol. 29, no. 1, pp. 91-99, 2001.

[15] L. P. Mayer, N. A. Pearsall, P. J. Christian et al., "Longterm effects of ovarian follicular depletion in rats by 4-vinylcyclohexene diepoxide," Reproductive Toxicology, vol. 16, no. 6, pp. 775-781, 2002.

[16] P. J. Devine, I. G. Sipes, M. K. Skinner, and P. B. Hoyer, "Characterization of a rat in vitro ovarian culture system to study the ovarian toxicant 4-vinylcyclohexene diepoxide," Toxicology and Applied Pharmacology, vol. 184, no. 2, pp. 107-115, 2002.

[17] I. Merchenthaler, M. V. L. Min Zhan, and P. B. Hoyer, "The 4-vinylcyclohexene diepoxide (VCD)-treated rat provides a unique preclinical model to study peri- menopausal hot flushes," Journal of Endocrinology, Diabetes \& Obesity, vol. 2 (2): 1028, 2014. 
[18] E. Y. Kim, I.-H. Baek, and M. R. Rhyu, "Cardioprotective effects of aqueous Schizandra chinensis fruit extract on ovariectomized and balloon-induced carotid artery injury rat models: effects on serum lipid profiles and blood pressure," Journal of Ethnopharmacology, vol. 134, no. 3, pp. 668-675, 2011.

[19] X.-Y. Wang, Z.-L. Yu, S.-Y. Pan et al., "Supplementation with the extract of schisandrae fructus pulp, seed, or their combination influences the metabolism of lipids and glucose in mice fed with normal and hypercholesterolemic diet," Evidence-Based Complementary and Alternative Medicine, vol. 2014, Article ID 472638, 11 pages, 2014.

[20] J. S. Kang, M. H. Han, G.-Y. Kim et al., "Nrf2-mediated HO-1 induction contributes to antioxidant capacity of a schisandrae fructus ethanol extract in C2C12 myoblasts," Nutrients, vol. 6, no. 12, pp. 5667-5678, 2014.

[21] Y. Xu, Z. Liu, J. Sun et al., "Schisandrin B prevents doxorubicininduced chronic cardiotoxicity and enhances its anticancer activity in vivo," PLoS ONE, vol. 6, no. 12, Article ID e28335, 2011.

[22] A. Panossian and G. Wikman, "Pharmacology of Schisandra chinensis Bail.: an overview of Russian research and uses in medicine," Journal of Ethnopharmacology, vol. 118, no. 2, pp. 183-212, 2008.

[23] Y. Takimoto, H.-Y. Qian, E. Yoshigai, T. Okumura, Y. Ikeya, and M. Nishizawa, "Gomisin N in the herbal drug gomishi (Schisandra chinensis) suppresses inducible nitric oxide synthase gene via $\mathrm{C} / \mathrm{EBP} \beta$ and $\mathrm{NF}-\kappa \mathrm{B}$ in rat hepatocytes," Nitric OxideBiology and Chemistry, vol. 28, pp. 47-56, 2013.

[24] J. del Pino, E. Martín-Gómez, M. Martín-Rodríguez et al., "Influence of sex, age, and menopause in serum osteocalcin (BGP) levels," Klinische Wochenschrift, vol. 69, no. 24, pp. 11351138, 1991.

[25] S.-Z. Liu, L.-F. Tian, P. Xu et al., "Analysis of correlation between blood biochemical indicators and bone mineral density of postmenopausal women," Molecular Biology Reports, vol. 38, no. 2, pp. 939-948, 2011.

[26] M. J. Romero-Aleshire, M. K. Diamond-Stanic, A. H. Hasty, P. B. Hoyer, and H. L. Brooks, "Loss of ovarian function in the VCD mouse-model of menopause leads to insulin resistance and a rapid progression into the metabolic syndrome," American Journal of Physiology-Regulatory Integrative and Comparative Physiology, vol. 297, no. 3, pp. R587-R592, 2009.

[27] L. A. Latiff, S. Parhizkar, M. A. Dollah, and S. Tajuddin Syed Hassan, "Alternative supplement for enhancement of reproductive health and metabolic profile among perimenopausal women: a novel role of Nigella sativa," Iranian Journal of Basic Medical Sciences, vol. 17, no. 12, pp. 980-985, 2014.

[28] C. A. Finn, "Reproductive ageing and the menopause," The International Journal of Developmental Biology, vol. 45, no. 3, pp. 613-617, 2001.

[29] S. Titus, F. Li, R. Stobezki et al., "Impairment of BRCA1-related DNA double-strand break repair leads to ovarian aging in mice and humans," Science Translational Medicine, vol. 5, no. 172, Article ID 172ra21, 2013.

[30] S. Hulley, D. Grady, T. Bush et al., "Randomized trial of estrogen plus progestin for secondary prevention of coronary heart disease in postmenopausal women," The Journal of the American Medical Association, vol. 280, no. 7, pp. 605-613, 1998.

[31] J. A. Cauley, D. G. Seeley, K. Ensrud, B. Ettinger, D. Black, and S. R. Cummings, "Estrogen replacement therapy and fractures in older women," Annals of Internal Medicine, vol. 122, no. 1, pp. 9-16, 1995.
[32] C. J. Kappeler and P. B. Hoyer, "4-vinylcyclohexene diepoxide: a model chemical for ovotoxicity," Systems Biology in Reproductive Medicine, vol. 58, no. 1, pp. 57-62, 2012.

[33] H. L. Brooks, D. P. Pollow, and P. B. Hoyer, "The VCD mouse model of menopause and perimenopause for the study of sex differences in cardiovascular disease and the metabolic syndrome," Physiology, vol. 31, no. 4, pp. 250-257, 2016.

[34] R. E. Nappi, E. Sinforiani, M. Mauri, G. Bono, F. Polatti, and G. Nappi, "Memory functioning at menopause: impact of age in ovariectomized women," Gynecologic and Obstetric Investigation, vol. 47, no. 1, pp. 29-36, 1999.

[35] J. I. Acosta, L. Mayer, J. S. Talboom et al., "Transitional versus surgical menopause in a rodent model: etiology of ovarian hormone loss impacts memory and the acetylcholine system," Endocrinology, vol. 150, no. 9, pp. 4248-4259, 2009.

[36] E. A. Cannady, C. A. Dyer, P. J. Christian, G. Sipes, and P. B. Hoyer, "Expression and activity of cytochromes P450 2E1, 2A, and $2 \mathrm{~B}$ in the mouse ovary: The effect of 4 -vinylcyclohexene and its diepoxide metabolite," Toxicological Sciences, vol. 73, no. 2, pp. 423-430, 2003.

[37] J. R. Haas, P. J. Christian, and P. B. Hoyer, "Effects of impending ovarian failure induced by 4 -vinylcyclohexene diepoxide on fertility in C57BL/6 female mice," Comparative Medicine, vol. 57, no. 5, pp. 443-449, 2007.

[38] L. T. Shuster, D. J. Rhodes, B. S. Gostout, B. R. Grossardt, and W. A. Rocca, "Premature menopause or early menopause: longterm health consequences," Maturitas, vol. 65, no. 2, pp. 161-166, 2010.

[39] J. C. Lohff, P. J. Christian, S. L. Marion, A. Arrandale, and P. B. Hoyer, "Characterization of cyclicity and hormonal profile with impending ovarian failure in a novel chemical-induced mouse model of perimenopause," Comparative Medicine, vol. 55, no. 6, pp. 523-527, 2005.

[40] R. T. Burkman, J. A. Collins, and R. A. Greene, "Current perspectives on benefits and risks of hormone replacement therapy," American Journal of Obstetrics and Gynecology, vol. 185, supplement 2, pp. S13-S23, 2001.

[41] V. Beral, "Breast cancer and hormone-replacement therapy in the Million Women Study," The Lancet, vol. 362, no. 9382, pp. 419-427, 2003.

[42] R. T. Chlebowski, S. L. Hendrix, R. D. Langer et al., "Influence of estrogen plus progestin on breast cancer and mammography in healthy postmenopausal women: the women's health initiative randomized trial," Journal of the American Medical Association, vol. 289, no. 24, pp. 3243-3253, 2003.

[43] E. Weiderpass, J. A. Baron, H.-O. Adami et al., "Low-potency oestrogen and risk of endometrial cancer: A case-control study," Lancet, vol. 353, no. 9167, pp. 1824-1828, 1999.

[44] J. E. Manson, J. Hsia, K. C. Johnson et al., "Estrogen plus progestin and the risk of coronary heart disease," The New England Journal of Medicine, vol. 349, no. 6, pp. 523-534, 2003.

[45] O. Wu, "Postmenopausal hormone replacement therapy and venous thromboembolism," Gender Medicine, pp. S18-S27, 2005.

[46] C. E. Dennehy, "The use of herbs and dietary supplements in gynecology an evidence-based review," Journal of Midwifery and Women's Health, vol. 51, no. 6, pp. 402-409, 2006.

[47] Y. B. Zhang, L. H. Wang, D. Y. Zhang, L. L. Zhou, and Y. X. Guo, "Ultrasound-assisted extraction and purification of schisandrin B from Schisandra chinensis (Turcz.) Baill seeds: optimization by response surface methodology," Ultrasonics Sonochemistry, vol. 21, no. 2, pp. 461-466, 2014. 
[48] M. Xu, G. Wang, H. Xie, Q. Huang, W. Wang, and Y. Jia, "Pharmacokinetic comparisons of schizandrin after oral administration of schizandrin monomer, Fructus Schisandrae aqueous extract and Sheng-Mai-San to rats," Journal of Ethnopharmacology, vol. 115, no. 3, pp. 483-488, 2007.

[49] J. L. Hancke, R. A. Burgos, and F. Ahumada, "Schisandra chinensis (Turcz.) Baill, Fitoterapia, vol. 70, no. 5, pp. 451-471, 1999.

[50] T. Yan, M. Xu, B. Wu et al., "The effect of Schisandra chinensis extracts on depression by noradrenergic, dopaminergic, GABAergic and glutamatergic systems in the forced swim test in mice," Food and Function, vol. 7, no. 6, pp. 2811-2819, 2016.

[51] P. Chen, S. Pang, N. Yang et al., "Beneficial effects of schisandrin $B$ on the cardiac function in mice model of myocardial infarction," PLoS ONE, vol. 8, no. 11, Article ID e79418, 2013.

[52] Y.-W. Choi, S. Takamatsu, S. I. Khan et al., "Schisandrene, a dibenzocyclooctadiene lignan from Schisandra chinensis: structure-antioxidant activity relationships of dibenzocyclooctadiene lignans," Journal of Natural Products, vol. 69, no. 3, pp. 356-359, 2006.

[53] R. A. Thandavarayan, V. V. Giridharan, S. Arumugam et al., "Schisandrin b prevents doxorubicin induced cardiac dysfunction by modulation of DNA damage, oxidative stress and inflammation through inhibition of mapk/p53 signaling," PLoS ONE, vol. 10, no. 3, Article ID e0119214, 2015.

[54] M.-K. Jang, Y.-R. Yun, S. H. Kim, J. H. Kim, and M. H. Jung, "Protective effect of gomisin N against endoplasmic reticulum stress-induced hepatic steatosis," Biological and Pharmaceutical Bulletin, vol. 39, no. 5, pp. 832-838, 2016.

[55] K. M. Ko, S. P. Ip, M. K. T. Poon et al., "Effect of a lignanenriched fructus Schisandrae extract on hepatic glutathione status in rats: protection against carbon tetrachloride toxicity," Planta Medica, vol. 61, no. 2, pp. 134-137, 1995.

[56] V. V. Giridharan, R. A. Thandavarayan, S. Sato, K. M. Ko, and T. Konishi, "Prevention of scopolamine-induced memory deficits by schisandrin B, an antioxidant lignan from Schisandra chinensis in mice," Free Radical Research, vol. 45, no. 8, pp. 950958, 2011.

[57] Z. Chen, M. Guo, G. Song et al., "Schisandrin B inhibits Th1/ Th17 differentiation and promotes regulatory $\mathrm{T}$ cell expansion in mouse lymphocytes," International Immunopharmacology, vol. 35, pp. 257-264, 2016.

[58] C. Y. Luo, L. Wang, C. Sun, and D. J. Li, "Estrogen enhances the functions of $\mathrm{CD} 4^{+} \mathrm{CD} 25^{+}$Foxp $3^{+}$regulatory $\mathrm{T}$ cells that suppress osteoclast differentiation and bone resorption in vitro," Cellular and Molecular Immunology, vol. 8, no. 1, pp. 50-58, 2011.

[59] X. L. Fan, X. B. Duan, Z. H. Chen, M. Li, J. S. Xu, and G. M. Ding, "Lack of estrogen down-regulates CXCR4 expression on Treg cells and reduces Treg cell population in bone marrow in OVX mice," Cellular and Molecular Biology, vol. 61, no. 2, pp. 13-17, 2015.

[60] N. E. Rance, P. A. Dacks, M. A. Mittelman-Smith, A. A. Romanovsky, and S. J. Krajewski-Hall, "Modulation of body temperature and LH secretion by hypothalamic KNDy (kisspeptin, neurokinin B and dynorphin) neurons: a novel hypothesis on the mechanism of hot flushes," Frontiers in Neuroendocrinology, vol. 34, no. 3, pp. 211-227, 2013.

[61] F. Kronenberg, "Menopausal hot flashes: a review of physiology and biosociocultural perspective on methods of assessment," Journal of Nutrition, vol. 140, no. 7, pp. 1380S-1385S, 2010.
[62] E. D. Szmuilowicz, C. A. Stuenkel, and E. W. Seely, "Influence of menopause on diabetes and diabetes risk," Nature Reviews Endocrinology, vol. 5, no. 10, pp. 553-558, 2009.

[63] C. J. Crespo, E. Smit, A. Snelling, C. T. Sempos, and R. E. Andersen, "Hormone replacement therapy and its relationship to lipid and glucose metabolism in diabetic and nondiabetic postmenopausal women: results from the Third National Health and Nutrition Examination Survey (NHANES III)," Diabetes Care, vol. 25, no. 10, pp. 1675-1680, 2002.

[64] A. Khan, M. Fortier, R. Reid et al., "Osteoporosis in menopause," Journal of Obstetrics and Gynaecology Canada, vol. 36, no. 9, pp. 839-840, 2014.

[65] W. Tiyasatkulkovit, N. Charoenphandhu, K. Wongdee, J. Thongbunchoo, N. Krishnamra, and S. Malaivijitnond, "Upregulation of osteoblastic differentiation marker mRNA expression in osteoblast-like UMR106 cells by puerarin and phytoestrogens from Pueraria mirifica," Phytomedicine, vol. 19, no. 13, pp. 11471155, 2012.

[66] A. García-Martín, M. María, I. Luque-Fernández, P. RozasMoreno, M. Quesada-Charneco, and M. Muñoz-Torres, "Osteocalcin as a marker of metabolic risk in healthy postmenopausal women," Menopause International, vol. 18, no. 5, pp. 537-541, 2011.

[67] B. L. Riggs, S. Khosla, and L. J. Melton III, "Sex steroids and the construction and conservation of the adult skeleton," Endocrine Reviews, vol. 23, no. 3, pp. 279-302, 2002. 


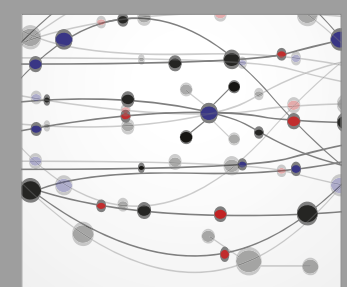

The Scientific World Journal
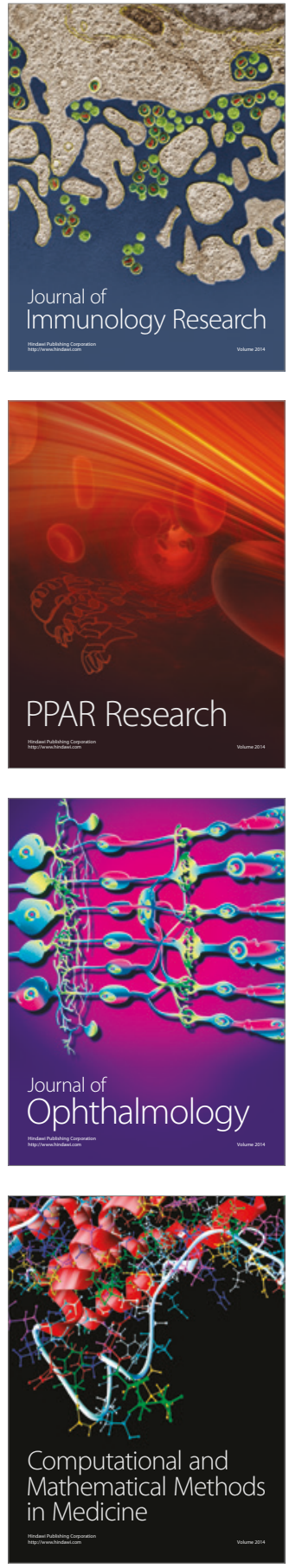

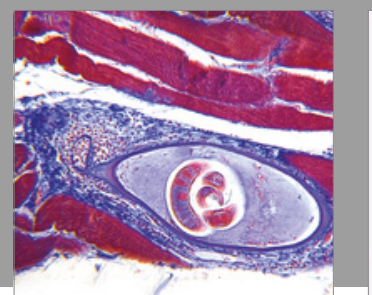

Gastroenterology Research and Practice
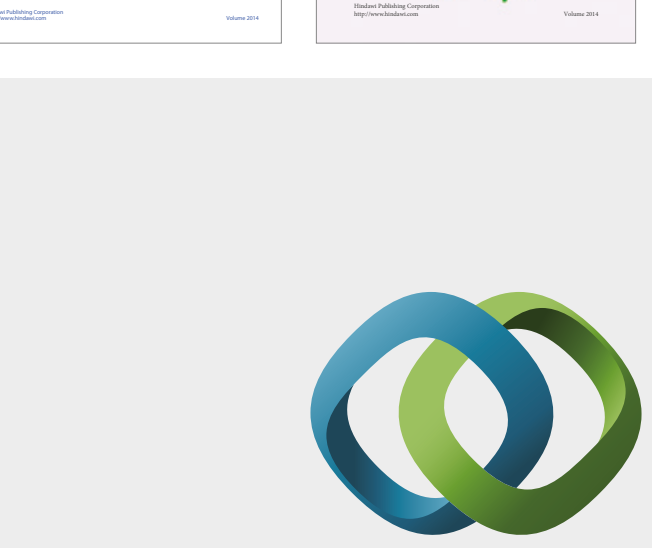

\section{Hindawi}

Submit your manuscripts at

https://www.hindawi.com
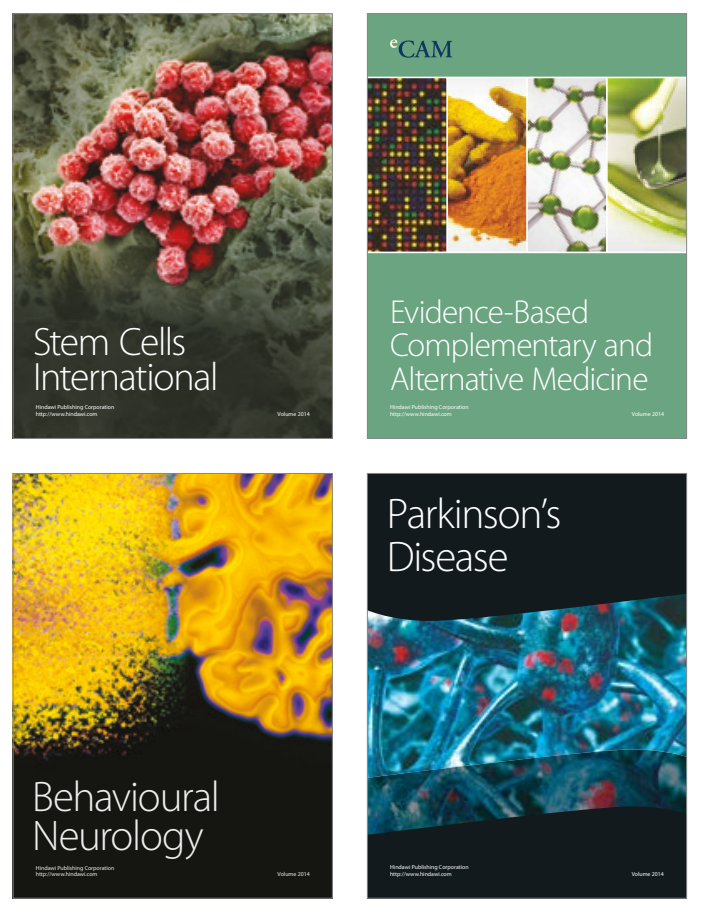
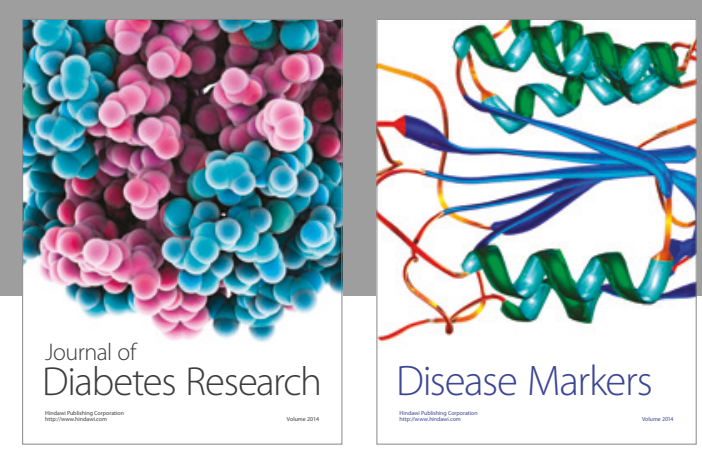

Disease Markers
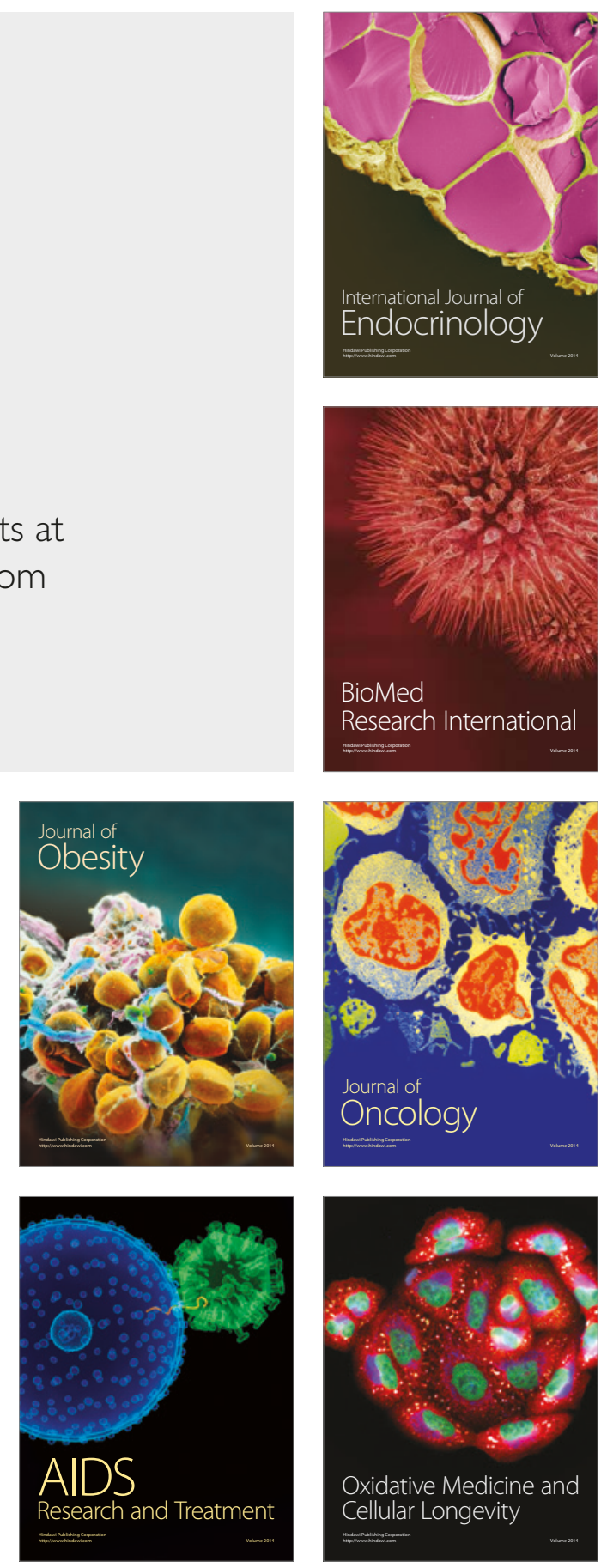